\title{
Circuit
}

Musiques contemporaines

\section{Harmonies : déserts fertiles que j'interroge}

\section{André Villeneuve}

Volume 10, numéro 1, 1999

Québec : génération fin de siècle

URI : https://id.erudit.org/iderudit/004706ar

DOI : https://doi.org/10.7202/004706ar

Aller au sommaire du numéro

Éditeur(s)

Les Presses de l'Université de Montréal

ISSN

1183-1693 (imprimé)

1488-9692 (numérique)

Découvrir la revue

Citer cet article

Villeneuve, A. (1999). Harmonies : déserts fertiles que j'interroge. Circuit, 10(1), 63-72. https://doi.org/10.7202/004706ar

\section{Résumé de l'article}

L'harmonie est l'élément clé sur lequel repose le processus créatif d'André Villeneuve. Il s'interroge, dans ce texte aux accents philosophiques et esthétiques, sur les différentes formes que peut prendre l’harmonie.
Ce document est protégé par la loi sur le droit d'auteur. L’utilisation des services d’Érudit (y compris la reproduction) est assujettie à sa politique d'utilisation que vous pouvez consulter en ligne.

https://apropos.erudit.org/fr/usagers/politique-dutilisation/ 


\title{
Harmonies : déserts fertiles que i'interroge
}

\author{
André Villeneuve
}

Déserts suppose ces lieux et notions visités et revisités, criblés par le questionnement, que l'on pourrait croire devenus stériles à force de fréquentations. Ces déserts, je les crois fertiles. En matière d'art, être sur le seuil du questionnement est nécessaire. Le contentement est sécheresse. II faut cerner l'idée de toute part, et, paradoxalement, être assiégé par elle. Ainsi, mon questionnement passe d'un registre à l'autre, en alternance, sans préséance et sans annonce d'un changement, de la théorie à la métaphore; tout simplement, le besoin de se délecter à la fois, dans un même élan, de la fleur et de la graine. Puis, dans une même foulée, le désir de passer de l'abstraction à la concrétude ', et inversement. Sans cesse d'une rive à l'autre sans le secours de catégories rassurantes. Ma réflexion n'est pas linéaire, elle ne l'a jamais été. D'ailleurs, je n'en connais aucune qui ne le soit: je pense aux lettres de Van Gogh, au journal de Delacroix, le paysage de leurs idées roule sur lui-même dans la foulée des doutes, esquisses et persistances. Ma réflexion a ses temples et ses refuges; sa géographie a ses plaines, ses cimes et certainement ses silences, sa toundra. Je procède par questions, je procède par phrases courtes: les déplacer comme une masse d'air. Mon travail illustre cette géographie, est déterminé par elle, secové par elle. J'aime le débordement contenu, l'excès délimité. J'ai toujours eu besoin de ces antagonismes, de cette apparente contradiction. Ils m'éclaicissent le sang, c'està-dire les idées. Ma réflexion est entêtée. Peut-être le signe d'une obsession de la transformation et de la gravité.

Journal du 29 juin 1995 :

Une idée, une seule, ne suffit-elle pas à une vie de musicien? Une lancée unique. En crever les bornes puis se laisser dériver vers de nouveaux rivages. La force active d'une idée est direction en elle-même. J'aime l'ivresse de la clarté et la lueur minimale d'une idée. Je ne me connais pas d'autres dévotions.

J'aime pivoter à partir de ce point. II me rattache au terrestre et, dans mes élans, absorbe quelque peu la chute conséquente puis, dans mes excès, me rappelle à la concision. "Veville la Transformation! criait Rilke. Sois enthousiaste de la flamme pour laquelle une chose te quitte en gloire de métamorphoses... » (Rilke, 1972, p. 161)

1. Par ce mot, nous entendons ici traduction, réalisation matérielle d'une idée. 


\section{Déserts fertiles}

Une réflexion se constitue. Elle ne s'établit pas d'elle-même dans l'isolement de la pensée suffisante. Sa croissance s'entrecoupe d'épisodes de digression, d'incursion vers d'autres voisinages, d'autres mondes où la pensée a trouvé son pain, amassé divers éléments et matériaux, où elle a fait sa récolte. Une quête d'outils et de savoir-faire, nourrie par la découverte d'autres territoires, musicaux, littéraires, picturaux, cinématographiques ou architecturaux. Dans mon esprit surgissent quelques noms: Flaubert, Saint-Augustin, Tarkovsky, et combien d'œuvres musicales. Ces quelques modèles furent et sont encore mes contrées, quelquesunes d'entre elles visitées par bonds successifs, d'autres que j'ai habitées. Ce voisinage d'autres mondes a nuancé, ciselé mon questionnement sur l'Harmonie. J'y ai ramassé mes galets; ils m'ont aidé à tracer le chemin de mes idées.

Tantôt il s'agissait "de nourrir sa petite symbolique personnelle » pour reprendre un mot de Ballif, tantôt "d'essayer d'en crever l'écran" d'un point de vue matériel, pour reprendre un mot de Boulez cité de mémoire. Un voyage d'artisan, guidé par l'alchimie des intuitions et la nécessité d'une quête, celle, si lourde de responsabilités en matière d'art, d'établir le territoire de son chant, et de fertiliser, par désir de cohérence avec Soi et quelques idées, ces déserts d'intentions qui nous habitent tous. Ce voisinage d'autres mondes, je le ramenais sous un même ciel, singulièrement, celuilà même à l'incipit de mon travail: comment a-t-on atteint, accédé à un tel degré de cohérence, entendons d'harmonie, dans son acception la plus large, entre ldée et Matériau?

Je pressentais bien que cette "cohérence " entrevue, perçue, n'était que le résutat d'une maîtrise technique, bien qu'elle soit indispensable, de l'usage du matériau propre à chacune des œuvres mais que ce même matériau lécrit, visuel ou sonore) reliait deux rives, tel un pont dressé entre l'abstraction, qui est le propre de I'Idée, et sa concrétude. En d'autres mots, ce que nous appellons Harmonie n'estce pas à la fois, cohérence du matériau avec lui-même, quel qu'il soit, et cohérence de ce matériau avec l'Idée? Cohérence qui est étymologiquement cohærens ou plutôt: "être soudé, attaché ensemble». Je reprends ma question. Harmonie, n'est-ce pas ce matériau soudé, rattaché à l'ensemble des moyens mis en œuvre pour la réalisation d'une idée, et à la fois ce même matériau investit par l'Idée? Je reviens en arrière. Je délimite le terrain, celui du musicien. 


\section{Harmonie}

Nous avons tous parcouru cette notion, sa grammaire musicale distinctive, sa nature, sa mécanique. Mais qu'en estil aujourd'hui, de cette notion? Peut-on la résumer et ne s'en tenir qu'à une mécanique de la langue musicale distinguant ainsi nos manières et esthétiques individuelles? Doiton l'aborder pour ensuite la rejeter essentiellement sur la rive de l'abstraction et de l'esthétique? Doit-on céder à un autre site, plus temporel, évolutif, cumulant des aspects divers, toujours renouvelés? Peut-on la résumer et ne s'en tenir qu'à un concept de proportions? La limiter à un système? La limiter à des catégories rassurantes qui comptabilisent manières, styles et frissonnent à la moindre exception? Grossière tendance, en pleine floraison autour de nous, qui réduit Harmonie à un système simpliste d'engrenages.

En ce sens, comment se fait-il que l'homme, qui a une propension à créer des systèmes afin de représenter, d'organiser et de gérer ce qu'il appréhende, en arrive à être prisonnier de ces derniers, tel un pêcheur dont les lignes s'entremêlent? L'immensité d'une telle notion nous fait-elle souffrir d'agoraphobie et nous pousse-t-elle alors aux excès de l'hégémonie? En matière d'art, toute réduction d'une œuvre à une mécanique ou à un système d'engrenages équivaut à priver d'eau une terre lors de son ensemencement. J'entends Mahler ou Schoenberg, Varèse, Tremblay ou Mather, je n'entends pas un système, mais une langue musicale qui sonne et dit. J'entends Harmonie. J'entends le «résonant» que le compositeur a ployé, organisé, tracé, en "désirs de cohérences avec ldée et Soi ».

Harmonie, mon questionnement. Des nuances aussi fertiles les unes que les autres se présentent à mon esprit comme autant d'avenues à emprunter. Faire sa récolte; délimiter une fois de plus le terrain de mes interrogations pour éviter le vertige; ramener cela à mon échelle de musicien. Je négocie une approche. Harmonies: Appels à la cohérence. Pour nous musiciens, appels à la cohérence dans le sonore et formes entre Idée et Objet. Échafauder une cohérence entre Idée et Objet, entendons par ce dernier, le matériau. N'est-ce pas là un des aspects fondamentaux de l'élaboration de nos manières de langage musical et de ses diverses grammaires? Je viens de réduire Harmonie à la notion de matériau, son acception la plus étroite, c'est-à-dire une manière d'organisation du sonore. Un pas de plus et je réduirai Harmonie à sa notion usuelle d'accords et d'enchaînements. Mais voilà qu'à cette échelle je rebondis de nouveau vers la notion de système — sans doute une part de l'héritage de l'enseignement reçu à un niveau élémentaire: système modal, tonal, dodécaphonique. Mais s'agitil de systèmes? Puis-je m'en tenir à cette seule notion en matière de composition? Tout système étant une réduction fonctionnelle pour une théorie, mais que les œuvres ont vite fait de distancer, ou plutôt de précéder. Même l'enseignement se satisfait parfois aveuglément de la notion de système: une première leçon d'harmonie ne 
devrait-elle pas en être une sur le timbre, éveiller l'oreille à l'agogique, au dynamisme du phénomène acoustique lui-même et non seulement porter sur l'identification d'un vocabulaire de base et la mémorisation de règles d'écriture? La notion de système est bien fonctionnelle, je la reconnais comme telle, mais à son tour elle me ramène vers une terre plus vaste.

Certes, quand je pense à Harmonie, je peux m'en tenir au vocabulaire, à une grammaire et à une syntaxe qui s'accordent à différentes manières, selon une époque ou une culture données, c'est-à-dire à ce que l'on appelle communément "style», mais en matière de composition, une autre dimension, plus précisément une autre étendue, une autre superficie de cette notion s'impose à mon esprit. Cette autre superficie n'estelle pas celle que nous parcourons chaque fois que nous arrachons I'ldée à son abstraction originelle et que nous l'acheminons vers la concrétude dans le sonore et dans le mouvement? Une lente traduction où notre désir de cohérence entre ldée et Matériau nous fait travailler à l'effort d'harmonie, j'entends, à l'effort de conserver - davantage qu'un lien logique entre ldée et Matériau - ce quelque chose d'impalpable, entre Soi et la poussée originelle, abstraite, de l'Idée. J'y reviendrai. Mais d'abord ceci : de l'Harmonie, j'essaie de repousser, pour un instant, l'aspect acoustique. Mise de côté salutaire afin de mieux y revenir, une fois de plus, nourri par d'autres incursions et cela, toujours dans le but de faire sa récolte.

Passons à l'aspect suivant, immatériel cette fois. Harmonie est affinité et union, concordia, concentus. Harmonie est aussi quête qui se traduit par "éthique» souhaits de cohérence avec Soi. Harmonie, dans cette acception, devient lieux acceptés de nos diversités et de nos contradictions. De façon plus nuancée : elle devient lieux d'évolution et de doutes, reliés par liens de conséquences et d'aboutissements toujours mis en péril de recommencement. Une quête d'harmonie dans la distinction et le mélange. J'insiste sur ce dernier mot. Une quête d'union et de compréhension, idéalement, sans niveler la nature distinctive de ce qui nous entoure. Je pense à Marc-Aurèle. Je pense à Lucrèce et à son De rerum natura. Je pense aussi à une autre voie, en opposition à la précédente, celle-ci se traçant par le nivellement de cette nature distinctive: Saint-Augustin, tel qu'il s'est présenté dans ses Confessions et, singulièrement, quelque 1500 ans plus tard, Wittgenstein. Leur point commun: une quête humaniste dans l'appel des proportions que peut prendre la volonté de compréhension, la volonté de cohérence entre l'Homme et le Monde. Une quête humaniste, "la leçon de vie», son expression matérielle par l'écrit, l'architecture, la couleur, le sonore, son expression immatérielle, spirituelle ou plus aérienne, par la parole. Harmonie m'apparaît alors comme une enveloppe temporelle idéalisée. Harmonie devient conciliation recherchée entre l'Homme et le Monde, les faits et les choses qui l'entourent.

Je regagne, maintenant, de l'harmonie, son aspect matériel. Je me tiendrai loin du cours d'histoire mais qu'on me permette, à l'occasion, quelques raccordements avec celle-ci et que l'on conserve en mémoire l'état (au sens ontologique) de ce qui a été dit précédemment, car c'est à partir de cette glaise que j'ai 
façonné mon harmonie matérielle en tentant de la rattacher par liens de conséquences aux leçons que l'on tire de la pratique musicale et, en toute humilité, de l'histoire, autant celle de mes contemporains que celle des siècles passés.

Harmonie matérielle est histoire de maçonnerie. Une digression. De nouveau un bond de la pensée vers la généalogie du mot. Harmonia est aussi, étymologiquement, "cheville, joint». De l'idée d'assemblage, d'ajustement, issue de la racine indo-européenne are qui nous donna "art», de l'idée, dis-je, d'assembler, d'ajuster, d'accorder. Mon imagination sur le seuil de l'aventure. Harmonie serait-elle histoire d'ajustements, de jonctions, d'accords (dans le sens ici de raccordements)? Delacroix cherchant la couleur juste. Flaubert, Mallarmé, le phonème juste, l'euphonie, c'est-à-dire, "l'harmonie des sons dans le mot». Une autre digression. Un autre bond, à caractère plus ludique cette fois. Pour un instant, représentons-nous, chacun, quelques regroupements, quelques manières d'harmonies propres à notre oreille. Tentative pour délimiter le terrain, circonscrire le sujet, esquisse d'ordre du parcours et des bonds de la pensée.

Pour ma part, je propose ces regroupements:

Harmonie matérielle. Dans son acception la plus large selon la nature du phénomène: toute donnée matérielle, purement et simplement; toute réunion de phénomènes sonores quels qu'ils soient.

Harmonie d'euphonie (assonances, allitérations). Beethoven disait de ses contrepoints à trois voix: " ll faut chercher la plénitude des rapports. " Les immenses étalements d'accords de trois sons dans certains des Quatre derniers lieder de Richard Strauss. Flaubert, le début de Salammbô : les sonorités ouvertes des « a » modulant vers celles, sifflantes, des «s " puis, modulant de nouveau vers celles, labiales, des «b»:

C'était à Mégara, faubourg de Carthage, dans les jardins d'Hamilcar.

Les soldats qu'il avait commandés en Sicile se donnaient un grand festin pour célébrer le jour anniversaire de la bataille d'Eryx, et comme le maître était absent et qu'ils se trouvaient nombreux, ils mangeaient et ils buvaient en pleine liberté.

(1964, p. 28)

Autres exemples: accord de timbre (Schoenberg, op. 5, n 5 ; Berg, Debussy, Varèse). Plus près de nous, bien des pages et certains mobiles de Gilles Tremblay — particulièrement ceux qui joignent à l'annulation de l'effet de "gravitation », créé par l'absence de métrique (ce qui est l'essence du mobile), une sonorité et une harmonie d'ensemble. Précisément ceux (les mobiles) où l'on trouvent des rapports harmoniques simples et équilibrés par l'orchestration entre une fondamentale et ses premiers sons harmoniques: ces moments sont de véritables colonnes de sons, solitaires, en rimes harmoniques. Un singulier rapprochement à faire, ici, avec cette autre colonne de sons qu'est le début de la Neuvième de Beethoven. À une tout autre échelle, que je n'hésiterais pas un instant à qualifier d' "océanique", un autre 
type d'harmonie d'euphonie: le continuum, masse sonore roulant sur elle-même, modulée par elle-même. C'est ainsi que j'entends la fin d'Oralléluiants (1975), pour nommer une fois de plus Gilles Tremblay. Certes, techniquement, un mobile ${ }^{2}$, mais par sa durée, son immense déploiement, un continuum.

Échelle-harmonie. Ici, c'est la pérennité du mode, sa flexibilité.

Harmonie comme "mot». Le moto qui est, dans la plupart des cas, accord de fonction agogique: les grands développements agogiques de Beethoven, Brahms, Schoenberg, Varèse, Debussy, Boulez.

Harmonie synesthésique. Messiaen, accords de couleurs. L'œil et l'oreille.

Harmonie phonétique. Tremblay, Fleuves: les "accords-traductions» des mots "fleuve", "soleil ", "cloches», "fleurs" par exemple.

Harmonie hiératique. Où les éléments sont fixes, essentiellement sculptés par le rythme. Bartók, de nombreux exemples. Stravinski, ces fameuses dominantes superposées du Sacre. Harmonie immuable sculptée par l'accentuation rythmique.

Je m'arrête ici, et laisse votre imagination faire ses parcours; votre oreille, s'ajuster à d'autres règles de jeu. Une question pourtant. De nouveau la même heurtant mon esprit: "Ma représentation de ces mondes harmoniques peut-elle me servir? »Loin de la définition, comment me représenter ces mondes harmoniques, ces manières d'harmonies, ces manières de sons, de regroupements, comment les souder et les rattacher — sorte de théorie des ensembles - par leurs distinctions et leurs zones communes pour que cela me serve en matière de composition? Unique but. Ramasser ces galets, mais construire, éviter la simple collection. Pour que cela me serve, sans intention de systèmes, mais dans un désir de cohérences avec Soi et quelques idées.

Harmonie ne peut se résumer qu'à un concept d'affinité. Ne peut exclure l'idée d'opposition, d'antagonisme, de différence. Harmonie suppose aussi la "division", la désunion; elle est fruit des coïncidences ou de la fréquentation, étudiée ou non, organisée ou non, de matériaux divers. Eł l'étymologie ne gouverne pas tout, mais nous montre une filiation au cours du temps. Revenons en arrière. Je négocie une autre approche.

Plus tôt j'ai dit: " [...] de l'action d'assembler, d'ajuster, celle d'accorder, c'est-àdire, de mettre en accord, de joindre". Cette question alors: "Qu'en est-il dans la résonance? » Accord suppose la jonction de deux éléments ou de divers éléments. L'accord est réunion. Matériellement, à l'origine, la symphonia des Grecs, la quinte, un simple rapport d'intervalle, symphonia signifiant «le mélange parfait entre deux sons ", l'Accord "parfait», exprimant quant à lui un rapport harmonique simple entre ces sons. Je pense aussi au dynamisme acoustique de ce mélange originel.
2. Voir en page 15 la définition que propose Marc Hyland. [NDLR] 
Je pense aussi à la "diaphonie» (qui nous donne la notion de di-sonance) et à son action agogique. L'énorme envolée depuis Pythagore, où "accord" est échelle jusqu'au moment où il se dresse dans sa verticalité élémentaire. Puis je pense à Rameau, où l'accord est don de la résonance, ainsi constitué dans son acception moderne, expression synthétique et hiératique d'une portion de l'échelle acoustique. Enfin, son expansion jusqu'à nous révélée par un phénomène d'addition, de division d'agglomération et d'hétérogénéité. Quelle est ma lecture, mon entendement de cela? Non pas un désir de situer historiquement quelques idées mais une volonté de cohérence, de regroupement de ces parallélismes, de ces juxtapositions, de l'horizon le plus éloigné au point le plus proche de soi. Sans doute en "gloire de sens" pour paraphraser Rilke.

\section{Harmonie est conciliation}

Conciliation recherchée entre les caractères de l'Idée et ceux, acoustiques, du résonant. Conciliation de l'idée, de son abstraction, de son état de perpétuelle "flottaison » et de sa «mise en durée », de sa "mise en rythme » dans le résonant. Harmonie est architecture de la traduction de l'Idée, la conciliation dans la diversité, le multiple et le mélange. En d'autres mots, concilier par Harmonie, par le résonant, ou le "sonore particulier à une œuvre" dirait Claude Ballif, concilier, dis-je, par le résonant, la diversité des avenues empruntées lors de la mise en route de l'Idée depuis son abstraction jusqu'à sa concrétude. Conciliation entre racines et feuillages. Dans le résonant, joindre, souder ces deux rives. Dans le résonant, dans la division du résonant - matériau concret — s'accorder à l'ldée, matériau abstrait. La division du résonant? Simplement, le grain de la résonance, c'est-à-dire ses harmoniques constitutives, ses intervalles. L'intervalle unit, distingue, divise. L'intervalle étant l'instrument dynamique de la division du résonant, de la division de l'Accord. Accord étant pris ici, une fois de plus, dans son acception la plus large: résonance, échelle ou agrégation.

Dans ce questionnement sur cette mécanique de l'intervalle, je me remémore les cours d'analyse de Ballif sur les lieder de Webern. L'intervalle comme "référentiel», selon son expression. Je renvoie ici à son ouvrage Économie musicale. De l'intervalle, considérer aussi son aspect brut, statique: une délimitation élémentaire: puis-je penser l'intervalle comme du silence délimité ? L'intervalle n'estil pas aussi une borne? L'intervalle n'est-il pas aussi un signe, une signature: Wagner, Berg?

Enfin, quelle est ma récolte? OEuvrer et se rattacher à la finitude dans la reconnaissance de la matière. Ma conviction que l'élan humaniste ne peut se faire que dans la réconciliation avec celle-ci, la matière et ses limitations. Mon travail est 
un essai de réconciliation avec ce poids: la permanence dans la répétition, la permanence dans la variation.

Journal du 18 juillet 1996 :

Faire son temple est évolution. La nécessité de remplir et faire, dans la division, face à notre propre finitude, notre propre fin de matière. OEuvrer est aussi l'imposition de la douceur à l'idée de cette réconciliation. Et, depuis celle-ci, le désir de s'y arracher une liberté.

Très succinctement voici cette généalogie de mon parcours: d'abord il y eut la préséance de l'intervalle, puis de l'échelle, enfin l'Accord s'est constitué, s'est dressé conséquemment à la polyphonie. Puis, il y eut le Choral, une réunion des précédents - expression hiératique de l'Accord, c'est-à-dire, l'Accord comme assise, comme formant et non comme un simple événement surgissant dans le cours d'une écriture polyphonique et, finalement, le Tracé. Le Tracé comme abstraction de l'idée de choral.

Ces plateaux caractéristiques: premières approches de la notion d'accord et du champ harmonique de la résonance dans Deuxième Terre pour piano (1985). L'Accord, quel profil a-t-il ? En fait, il est le résultat, d'une part, de la superposition des intervalles-formants de l'œuvre (secondes et tierces), et d'autre part, de la coïncidence travaillée, orientée, sculptée des diverses parties de la polyphonie. Puis à un moment très précis, dans le parcous de la forme, il surgit et repose sur une fondamentale clairement exprimée.

l'Accord, dressé dans sa verticalité, "don de la résonance", s'enracinerait dorénavant sur une fondamentale ou deux et ne serait plus le résultat d'agrégat d'intervalles. Ce fut là mon "pont pivotant», celui à partir duquel ma syntaxe s'est élaborée. Dorénavant la structure harmonique d'un accord gouvernerait, à son tour, celle de l'œuvre. La structure verticale (intervalles constitutifs) de l'Accord ou des accords de base serait projetée horizontalement et, déterminerait ainsi les progressions harmoniques. En d'autres mots, les relations d'intervalles, d'une fondamentale à l'autre, se trouvent issues et rattachées à celles du matériau harmonique d'origine. 


\section{Usage du Choral comme installation de l'Accord (1991-1995) \\ Le Tracé, comme abstraction du Choral (1996-1999)}

L'accord, tout simplement, I'Accord comme colonnes de sons, l'Accord comme pilier, l'Accord et sa structure harmonique comme formant principal. Pourquoi le Choral ? Parce que j'en apprécie la tenue quasi hiératique de l'Accord, la réunion des parties, c'est-à-dire, plus précisément, la conciliation des intervalles constitutifs de l'Accord dans la modestie de leurs proportions, dans la force dynamique de leurs relations et, par-dessus tout, parce que i'y trouve la simplicité syllabique des rythmes et la portée du silence. Le Choral fut ma phrase courte en matière de composition: petit temple où rompre le silence, lui donner ses angles, son tracé. À la division de la forme en ces "autres lieux ou chorals » allait correspondre et se manifester une manière caractéristique de mon harmonie: la division de l'Accord.

l'Accord est un aménagement d'intervalles. Ce dernier, unique source du matériau harmonique, divisé en ses formants constitutifs et scindé aussi lorsque multiplié par lui-même. La coïncidence de ces portions d'accords, dont l'organisation harmonique cohérente repose sur des relations entre les notes de basses et/ou fondamentales réelles (tel que mentionné plus haut), constituera dans tous les cas ce que j'ai convenu d'appeler un cantus firmus structural.

Le Choral fut ma force tranquille, mon atome. Dans le temps et la forme contenue du Choral, j'aurai élaboré peu à peu une grammaire indispensable au déploiement de la force dynamique de mon harmonie au sein des Tracés solitaires d'humanité et du Concerto pour piano et orchestre à cordes (1997-1999). Les 29 Tracés solitaires d'humanité pour piano substituent, à l'intérieur de mouvements très contrastés, la variation thématique à la variation de formants harmoniques: harmonies caractérisées par leur regroupement, leur unité matérielle. Ces regroupements traités comme un bloc de marbre à sculpter, à mettre en mouvement: variations de leurs déploiements rythmique, agogique, mélodicoharmonique. Par opposition, les 17 Tracés solitaires d'humanité, pour chœur mixte a cappella, s'ancrent dans un matériau restreint, concis: harmonie traitée comme échelle, comme mode et sa multiplication. Le concerto, pour sa part, développe tous les aspects de la pièce pour piano précédemment citée et déborde sur la question du motif. Si l'Accord (l'Harmonie) caractérise une géographie des lieux harmoniques de l'Idée, le motif ne doit-il pas en caractériser le relief perceptible, délimité en ses territoires variés? 
Il y eut ces déserts, manières d'écritures à interroger, et il y a ceux à venir. Rien, dans la matière sonore, qu'elle-même, à l'exception de l'ldée venue la cerner. Céder à la matière, et parcourir l'Idée. Lui donner une "autre forme» par désir d'union avec la parole des hommes, et la nécessité de "faire» dans la durée. Je n'interroge pas le but, mais les directions suggérées du sonore. Je m'accorde la liberté de ses échos autant que le plaisir d'une fouille. Vie de l'homme, vie des idées, leurs poumons en commun. Harmonie est aussi parole.

BAllf, C. (1988), Économie musicale, Paris, Éditions Méridien Klincksieck.

Flaubert, G. (1964), Salammbô, Paris, Garnier-Flammarion.

RILKE, R. M., (1972), Sonnets à Orphée, Paris, Sevil. 